\title{
Twisted Poisson Structures and Non-commutatative/non-associative Closed String Geometry
}

\author{
Dieter Lüst* \\ Arnold Sommerfeld Center for Theoretical Physics \\ Department für Physik, Ludwig-Maximilians-Universität München \\ Theresienstr. 37, 80333 München, Germany \\ and \\ Max-Planck-Institut für Physik \\ Föhringer Ring 6, 80805 München, Germany \\ E-mail: dieter.luest@lmu.de, luest@mppmu.mpg.de
}

\begin{abstract}
In this paper we discuss non-commutative and non-associative geometries that emerge in the context of non-geometric closed string backgrounds. T-duality and doubled field theory plays an important role in formulating the corresponding effective action for these kind of non-geometric string backgrounds. As we will argue the emerging non-commutative and non-associative algebras for the closed string (dual) coordinates and (dual) momenta can be mathematically described by a twisted Poisson structure, in closed analogy to the phase space of a point particle moving in the field of a magnetic monopole.
\end{abstract}

Proceedings of the Corfu Summer Institute 2011 School and Workshops on Elementary Particle Physics and Gravity

September 4-18, 2011

Corfu, Greece

\footnotetext{
* Speaker.
} 
String flux compactifications (for reviews see e.g. see [1,2]) attracted a lot of interest during the recent years. In particular, they proved to be essential for moduli stabilization, they led to the discovery of a huge string flux landscape, and they are also relevant in the context of string inflationary scenarios in the early universe. Moreover, flux compactifications broadened the notion of geometry in string theory, since it became clear that generalized geometries, that are not geometric spaces, can still serve as consistent string backgrounds. Finally, T-duality also plays an important role within flux compactifications: background with and without $H$-fluxes can be T-dual to each other, and T-duality can transform a geometric space into a non-geometric background and vice versa. The so-called doubled geometry, where one includes the momentum coordinates together with the dual winding coordinates, turned out to be helpful to formulate an effective string action, which is invariant under T-duality transformations.

In any case, an extended string generically feels the background geometry rather different compared to a point particle. As it was discussed in the past by several authors, non-commutative geometry may naturally arise in string theory. In particular for open strings on the (2-dimensional) world volume of D-branes, their end-point coordinates become non-commuative when a constant $B$ - or $F$-field on the D-brane is turned on (see $[3,4,5,6]$ for a small selection of papers). In this background, the corresponding open string conformal field theory is still a free theory and hence exactly solvable. As a result of the CFT computation, the non-vanishing $B$ - or $F$-field induces a non-vanishing commutator of the open string end point coordinates of the form $(\mathscr{F}=B-F)$

$$
\left[X_{1}(\tau), X_{2}(\tau)\right]=\theta_{12} \quad \text { with } \quad \theta_{12}=-\frac{2 \pi i \alpha^{\prime} \mathscr{F}}{1+\mathscr{F}^{2}}
$$

Performing a T-duality transformation along one of the D-brane world volume coordinates, one obtains a geometric configuration with (1-dimensional) D-branes that intersect the coordinates axis in a certain angle, which is determined by the $B$ - or $F$-field Now, after the T-duality transformation, the dual open string coordinates are fully commuting.

This non-commutative open string algebra corresponds to a Poisson structure, which is similar to the Poisson structure of a point particle moving in a constant magnetic field, where however the non-commutativity shows up in the algebra of the momentum operators. By evaluating correlation functions of vertex operators in open string theory, it is possible to derive the Moyal-Weyl product. Then the algebra of the periodic functions can then be defined by an $N$-product $\star_{N}$ in the following way

$$
\begin{gathered}
f_{1}(x) \star_{N} f_{2}(x) \star_{N} \ldots \star_{N} f_{N}(x):= \\
\left.\exp \left(i \sum_{1 \leq n<m \leq N} \theta^{a b} \partial_{a}^{x_{n}} \partial_{b}^{x_{m}}\right) f_{1}\left(x_{1}\right) f_{2}\left(x_{2}\right) \ldots f_{N}\left(x_{N}\right)\right|_{x_{1}=\ldots=x_{N}=x}
\end{gathered}
$$

These $N$-products are related to the subsequent application of the usual star-product $\star=\star_{2}$ in the following way

$$
f_{1} \star_{N} f_{2} \star_{N} \ldots \star_{N} f_{N}=f_{1} \star f_{2} \star \ldots \star f_{N} .
$$

Here we are investigating the geometry of closed strings moving in an $H$-field background and its T-dual versions. This is a more difficult problem, since non-vanishing $H$-field implies that the $B$ field is non-constant and hence the corresponding two-dimensional $\sigma$-model is not anymore exactly 
solvable. The deformation from the flat space by the $H$-field flux or, respectively, by its T-dual metric components imply that we necessarily have to consider at least three-dimensional backgrounds in order to obtain closed string non-commutativity, whereas open string non-commutative geometry already arises in two dimensions. In fact, the spaces we will consider are twisted, there-dimensional tori, where a two-torus is non-trivially fibred over a circle in the third direction [7, 8, 9]. In the first example one starts with a flat three-torus with constant $H$-field. Here the $B$-field on the fibred two-torus is linear in the circle coordinate. The corresponding monodromy transformations, called parabolic monodromies, on the fibred two-torus are of infinite order, when transporting it along the base circle. As we will explain in more detail in the following, there exists a chain of three T-duality transformations starting with the $H$ flux, leading to four different types of geometrical and non-geometrical fluxes:

$$
H_{a b c} \stackrel{T_{a}}{\longrightarrow} f_{b c}^{a} \stackrel{T_{b}}{\longrightarrow} Q_{c}{ }^{a b} \stackrel{T_{c}}{\longrightarrow} R^{a b c} .
$$

Here $T_{a}$ denotes T-dualizing along direction $a$ and $H$ is a three-form. The $f$ 's are called geometric fluxes, and they are given by the first derivatives of the vielbein and are related to the Levi-Civita spin connection and therefore to the curvature of the twisted torus (Nil-manifold). On the other hand, and the $Q$ 's and the $R$ 's are non-geometric fluxes and their geometric meaning will be discussed later in this paper.

As we will discuss in section three, for these kind of spaces, the closed string geometry is non-commutative and also non-associative [10,11, 12, 13, 14, 15]. First, consider the commutator of the (dual) coordinates on the fibred two-torus. For non-vanishing fluxes $F$ they do not commute,

$$
\left[X^{1}, X^{2}\right] \simeq F p^{3}
$$

Depending on the given duality frame, the fluxes $F$ correspond to either $F=H, f, Q$ or $R$-flux, respectively. The coordinates $X^{1}, X^{2}$ are either coordinates or dual coordinates on the fibred twotorus, again depending on the considered duality frame. Finally, $p^{3}$ is the momentum or the dual momentum in the third circle direction. Second, the three-bracket between all three closed string coordinates or dual coordinates is non-vanishing:

$$
\left[X^{1}, X^{2}, X^{3}\right] \simeq F
$$

This result shows that the $H, f, Q, R$-deformed closed string geometry exhibits not only non-commutative but even genuine non-associative structures.

Eqs.(2.21) and (3.21) together with $\left[X^{k}, p^{k}\right]=i$ now define a so-called twisted Poisson structure. As discussed in [16] (same proceedings), this algebra can be nicely described by quantizing 2-plectic manifolds in loop space using groupoids. Moreover, this structure also emerges for the momenta of point particles moving in the field of a magnetic monopole, where again the role of coordinates and momenta is exchanged compared to the closed string geometry. We will argue that in closed string theory the emergence of this twisted Poisson structure is a stringy feature, related to the fact that the closed string can move in non-geometric string backgrounds. In particular, since T-duality is relating left-right symmetric backgrounds to left-right asymmetric closed string backgrounds, the non-commuative and non-associtive geometries in particular arise for the coordinates 
of the left-right asymmetric spaces. In this sense, the $R$-flux background is left-right asymmetric in all three world-sheet string coordinates.

For the case of constant $H$-resp. $R$-flux, i.e. for the parabolic case, the underlying closed string CFT and its correlation functions were analyzed in [12]. The main result of this paper is that the algebra of periodic functions on these spaces, namely algebra of closed string (slighlty off-shell) tachyon vertex operators, becomes non-associative. It is given in terms of a new non-associative $N$-product

$$
\begin{gathered}
f_{1}(x) \Delta_{N} f_{2}(x) \Delta_{N} \ldots \Delta_{N} f_{N}(x) \stackrel{\text { def }}{=} \\
\left.\exp \left[\frac{\pi^{2}}{2} F^{a b c} \sum_{1 \leq i<j<k \leq N} \partial_{a}^{x_{i}} \partial_{b}^{x_{j}} \partial_{c}^{x_{k}}\right] f_{1}\left(x_{1}\right) f_{2}\left(x_{2}\right) \ldots f_{N}\left(x_{N}\right)\right|_{x},
\end{gathered}
$$

which is the closed string generalization of the open string non-commutative product eq.(2). This completely defines the new tri-product, which satisfies the relation

$$
f_{1} \triangle_{N} f_{2} \triangle_{N} \ldots \Delta_{N} f_{N-1} \Delta_{N} 1=f_{1} \Delta_{N-1} \ldots \Delta_{N-1} f_{N-1} .
$$

Specializing above expression to $N=3$ gives

$$
\left.f_{1}(x) \triangle f_{2}(x) \triangle f_{3}(x) \stackrel{\text { def }}{=} \exp \left(\frac{\pi^{2}}{2} \theta^{a b c} \partial_{a}^{x_{1}} \partial_{b}^{x_{2}} \partial_{c}^{x_{3}}\right) f_{1}\left(x_{1}\right) f_{2}\left(x_{2}\right) f_{3}\left(x_{3}\right)\right|_{x},
$$

Besides the parabolic case, also twisted, there-dimensional tori with elliptic monodromy properties of the fibre two-torus can be studied. As we will discuss in the last section, here the elliptic monodromies are of finite order $N$, which means that the fibre two-torus comes back to itself when transported $N$-times around the circle base. T-duality again maps a geometric background to a non-geometric $Q$ and $R$-flux background, which is non-commutative and non-associative. ${ }^{1}$ The corresponding fluxes are not anymore constant but given in terms certain modular functions of the base coordinates. Hence at first sight, it seems hard to argue that these spaces correspond to consistent conformal string backgrounds, valid to all order in $\alpha^{\prime}$. However it was shown [14] that at a particular point in moduli spaces, non-geometric backgrounds with elliptic monodrmies can be formulated as exact CFT's, namely as symmetric or asymmetric freely acting orbifolds, depending on whether one is dealing with a geometric or non-geometric closed string background. For these (a)symmetric orbifold spaces the full modular invariant partition function can be constructed, and the non-commuative behavior of the closed string coordinates follows as an exact CFT result in the (a)symmetric orbifold limit.

\section{Twisted Poisson structures for point particles}

In this section we first compare the non/commutative and non-associative closed string algebra, displayed in eqs.(2.19) and (7), with the phase space structure of a particle moving a magnetic field. It turn out the the closed string case corresponds to a twisted Poisson structure algebra, discussed in $[17,18,19,20]$, of a point particle moving in the spatially extended, non-constant magnetic field of a magnetic monopole. However going from the point particle to the closed string as a probe of

\footnotetext{
${ }^{1}$ The closed string non-commutativity in relation with T-duality and non-geometric versus geometric string background was discussed for the first time in a model with elliptic $Z_{4}$-monodromy in [11].
} 
the target space geometry, momentum and position operators are exchanged. A similar application applies for a point particle moving in a constant magnetic field as compared to the motion of an open string in the B- and F-filed background described at the beginning: whereas for the point particle the position operators $X_{i}$ do commute, whereas the (mechanical) momentum operators do not commute, in the open strings the position operators become non-commuting. In both cases, for open and for closed strings, the exchange the non-commutatativity and non-assoiativity in the closed string $X_{i}$-coordinates is due to the contribution of the stringy oscillators, as we will discuss in the specific example.

\subsection{Point particle in a constant magnetic field}

Let us first consider a charged point particle which is moving in a magnetic field $\vec{B}$. The configuration space $\mathscr{M}$ is the tangent bundle of some manifold $\mathscr{Q}$ :

$$
\mathscr{M}=T^{*} \mathscr{Q} .
$$

The Langrange function of the particle (we sets its mass and its charge equal to one) with coordinate $x^{i}(t)$ is given by the follow expression

$$
L=\frac{1}{2}\left(p_{i}\right)^{2}=\frac{1}{2}\left(\dot{x}^{i}-A^{i}\right)^{2},
$$

where $\vec{A}(x)$ is the vectorpotential and $\vec{B}=\operatorname{rot} \vec{A}$. The canonical momenta $p_{i}=\frac{\partial L}{\partial \dot{x}^{i}}=\dot{x}^{i}-A^{i}$ satisfy the standard, canonical Poisson algebra:

$$
\pi^{i j}=\left\{x^{i}, x^{j}\right\}=0, \quad\left\{p_{i}, p_{j}\right\}=0, \quad\left\{x^{i}, p_{j}\right\}=\delta_{i}^{j} .
$$

However this is not anymore true, when we consider the algebra of the mechanical momenta $\bar{p}^{i}=$ $\dot{x}^{i}=p^{i}+A^{i}$. Consider e.g. the simplest case of a 2-dimensional base manifold $\mathscr{Q}$ with a linear vector potential $\vec{A}$, i.e with a constant magnetic field $\vec{B}$. Then one obtains after a short calculation the following Poission algebra:

$$
\pi^{i j}=\left\{x^{i}, x^{j}\right\}=0, \quad \pi_{i j}=\left\{\bar{p}_{i}, \bar{p}_{j}\right\}=\varepsilon_{i j k} B^{k}, \quad\left\{x^{i}, p_{j}\right\}=\delta_{i}^{j} .
$$

Hence, for a particle moving in a constant magnetic field the mechanical momenta do not commute anymore, but their commutator is given by the constant magnetic field. As its is well known, this form of non-commtatative algebra occurs for the open string coordinates on D2-branes moving in the background of a constant magnetic field. Note that comparing point particles with the open string, the role of momenta and positions are essentially exchanged.

\subsection{Point particle in the field of a magnetic monopole}

Now we consider a base manifold $\mathscr{Q}$, which is at least 3 -dimensional. The $B$-field is non anymore assumed to be constant and can be even topologically non-trivial:

$$
\vec{B} \in H^{2}(\mathscr{Q})
$$

Therefore we allow the possibility that $B$ is non-closed:

$$
H=d B=\star \rho_{\text {magn }} .
$$


In three dimensions, $\rho_{\text {magn }}$ is the magnetic charge density of a magnetic monopole, which is smeared out over space. Let us again assume the simplest case that the 3 -form $H$ is constant over space, i.e. the magnetic field $\vec{B}$ is a spatially linear 2 -form. This implies the following new Poisson algebra:

$$
\pi^{i j}=\left\{x^{i}, x^{j}\right\}=0, \quad \bar{\pi}_{i j}=\left\{\bar{p}_{i}, \bar{p}_{j}\right\}=H_{i j k} x^{k}, \quad\left\{x^{i}, p_{j}\right\}=\delta_{i}^{j} .
$$

In addition, using the still valid canonical relation $\left\{x^{i}, p_{j}\right\}=\delta_{i}^{j}$, one discovers that this algebra is even non-associative. This means that Jacobi-identity among the mechanical momenta is not anymore satisfied, i.e. the double Poisson-bracket plus its permutations is non-vanishing:

$$
\bar{\pi}_{i j k}=\left\{\left\{\bar{p}_{i}, \bar{p}_{j}\right\}, \bar{p}_{k}\right\}+\text { perm. }=H_{i j k}
$$

This algebra is called twisted Poisson structure, it is non-commutative as well as non-associative with respect to the mechanical momentum variables. In this simplest case, the right hand side of the non-associative 3-bracket is a constant, namely just the exterior derivate of the magnetic field. As we will see in the following, the analogous twisted Poisson structure structure is present for closed strings coupled to a non-vanishing $H$-field background. However, the difference between point particles and the closed string is that coordinates and momenta will exchange their roles.

\section{Twisted Poisson structures from closed strings on non-geometric backgrounds}

\subsection{The T-dual chain of geometric and non-geometric flux backgrounds}

\subsubsection{Geometric flux backgrounds}

In the simplest case for constant background metric $g$ and $b$-field, $S O(d, d)$ T-duality transformations are just acting as automorphism on the moduli space of the string background space. However for non-constant background fields, T-duality transformations in general not only change the geometry of the associated background, but can rather lead to a topology change. This is in particular true for backgrounds with non-vanishing NS $H$-flux, i.e. where the $b$-field is non-constant. So for concreteness, let us consider the compactification on a three-dimensional torus $T^{3}$, which can be viewed as a $\mathscr{F}=T^{n}$ fibration $(n=0,1,2,3)$ over a (3-n)-dimensional base $\mathscr{B}=T^{3-n}$. In the following we will consider four different cases.

(Frame A) $n=0$ : The $H$-flux space:

Here there are $N$ units of constant, isotropic $H_{a b c}$-flux on $T^{3}$, i.e. $H=N d x \wedge d y \wedge d z$. Note, however, that the associated, non-constant $b$-field breaks the isotropy of the background, namely when writing it in a particular gauge as $b=N z d x \wedge d y$. In the following we will perform $T$-duality transformations over all directions of the $n$-dimensional $T^{n}$ fibre torus, and hence we discuss three additional background spaces [8, 9, 21]:

(Frame B) $n=1$ : The $f$-flux space:

Performing a T-duality transformation on the one-dimensional circle fibre $\mathscr{F}=T_{x}^{1}$ in $x$-direction, one obtains the Heisenberg nilmanifold, which is a twisted torus without $b$-field. It is topologically 
distinct from $T^{3}$, since it has $H_{1}(Z)=Z \times Z \times Z_{N}$. Its Levi-Civita connection can be related to the structure constants $f_{b c}^{a} \sim N$ of the Heisenberg group, where the

$$
f_{b c}^{a}=-2 e_{[b}^{m} e_{c]}^{n} \partial_{m} e_{n}^{a}
$$

are called geometrical fluxes. The effective action for the geometric string backgrounds of frames $\mathrm{A}$ and $\mathrm{B}$ is given in terms of the well-known low-energy supergravity action, its bosonic part is the standard Neveu-Schwarz (NSNS) Lagrangian:

$$
\mathscr{L}=e^{-2 \phi} \sqrt{|g|}\left(\mathscr{R}+4(\partial \phi)^{2}-\frac{1}{12} H_{i j k} H^{i j k}\right) .
$$

\subsubsection{Non-geometric flux backgrounds and doubled field theory}

(Frame C) $n=2:$ The $Q$-flux space:

Performing a T-duality transformation in the $x, y$-directions on the two-dimensional torus fibre $\mathscr{F}=T_{x, y}^{2}$, one obtains the so-called $Q$-space. This new background is again a $T^{2}$-fibration. But the corresponding metric and $b$-field are only locally defined, but not anymore globally. Hence this space is one of the simplest examples of a non-geometric string background that is not anymore a Riemannian manifold. The reason for the failure of being a Riemannian manifold is given by the observation that the fibre $\mathscr{F}$ has to be glued together by a T-duality transformation when transporting it once around the base $\mathscr{B}$, and not by a standard diffeomorphism. Concretely for our example, the Kähler structure $\rho=b+i \operatorname{Vol}\left(T^{2}\right)$ of $\mathscr{F}$ has the (parabolic) monodromy property

$$
\rho(z+2 \pi)=\frac{\rho(z)}{1+2 \pi N \rho(z)}
$$

which is just an infinite order element of the $T$-duality group $S O(2,2, Z)$. Here, the associated $Q$-flux shows up in eq.(2.3) as the discrete parameter, which determines the non-trivial modular transformation of $\rho(z)$.

The geometric role of the non-geometrtric fluxes can be nice described via the frame work of doubled field theory (DFT). DFT was introduced in [22, 23, 24, 25]. In this theory, T-duality is turned into a manifest symmetry by doubling the coordinates at the level of the effective space-time action for string theory. As already discussed in the introduction, T-duality relates momentum and winding modes of a closed string moving on a torus $T^{D}$ via the T-duality group $O(D, D)$. T-duality relates momentum and winding modes of a closed string moving on a torus $T^{D}$ via the T-duality group $O(D, D)$. When the coordinates are doubled, this duality symmetry can be made manifest. Thus, in DFT every conventional coordinate $x^{i}$, associated to momentum modes, is complemented by a dual coordinate $\tilde{x}_{i}$, associated to winding modes. The coordinates combine into a fundamental $O(D, D)$ vector $X^{M}=\left(\tilde{x}_{i}, x^{i}\right)$.

Specifically, consider the following field redefinition of the metric $g$ and the $b$-field:

$$
\left(\tilde{g}^{-1}+\beta\right)^{-1} \equiv \tilde{\mathscr{E}}^{-1}=\mathscr{E}=g+b,
$$

where we have introduced

$$
\tilde{\mathscr{E}}^{i j}=\tilde{g}^{i j}+\beta^{i j}
$$


where $\beta^{i j}$ is a bi-vector. We can also redefine $\phi$ :

$$
\sqrt{|g|} e^{-2 \phi}=e^{-2 d}=\sqrt{|\tilde{g}|} e^{-2 \tilde{\phi}} .
$$

The redefinition (2.4) has the form of an overall T-duality, here in all three toroidal directions. Following the recent work on the effective action of non-geometric fluxes [26, 27, 28, 29, 30], the $Q$-flux is now defined as

$$
Q_{m}{ }^{n k}=\partial_{m} \beta^{n k} .
$$

Note that, being a partial derivative of a bi-vector, $Q$ is not a tensor. However, as shown in [27, 30], the proper geometrical interpretation of $Q$ is playing the role of a connection, which allows us to construct a derivative for the dual $\tilde{x}$ coordinates that is covariant with respect to the $x$ diffeomorphisms. In addition one can show that for certain non-geometric situations, where the metric $g$ and the $b$-field are only locally but not globally defined, the $Q$-flux is nevertheless a globally welldefined object. In particular for the chain of T-duality transformations with constant flux, it turns out that $\beta^{i j}$ is linear in the circle coordinate $z, \beta^{x y}=N z$, and hence the $Q$-flux is simply given by $N$. i.e. $Q_{z}^{x y}=N$.

\section{(Frame D) $n=3$ : The $R$-flux space:}

Finally one can consider a T-duality transformation along the entire three-dimensional torus, being seen as a fibration with fibre $\mathscr{F}=T_{x, y, z}^{3}$ over a point. However this T-duality is much more problematic, since the $z$ direction is not any longer a Killing isometry of the background space. Hence the standard Buscher rules cannot be applied, and T-duality acts like a formal transformation in CFT, that leads to a "space" that is left-right asymmetric in all its three directions. This new background is not known explicitly, in fact it is not even locally a Riemannian manifold, describable by a metric $g$ and a $b$-field. However, as shown in [27,30], the associated $R^{a b c}$-flux can be again constructed in a geometric manner when using the formalism of doubled field theory. The reason, why this will be possible, is that in DFT T-duality transformations can be performed regardless if one is dealing with a Killing isometry or not. The $R$-flux can be viewed as the tensor, which is completely T-dual in all directions to the standard $H$-flux. Its precise definition is given by the following equation,

$$
R^{i j k}=3 \tilde{D}^{[i} \beta^{j k]},
$$

where we introduce the derivative operator

$$
\tilde{D}^{i} \equiv \tilde{\partial}^{i}-\beta^{i j} \partial_{j}
$$

This $R$ flux is a tensor and represents the covariant field strength of $\beta$. In the supergravity limit the DFT fields are taken to be independent of the dual coordinates, i.e. one sets $\tilde{\partial}^{i}=0$ in the action. Here the $R$ flux term is given as

$$
\left.R\right|_{\tilde{\partial}=0} ^{i j k}=3 \beta^{p[i} \partial_{p} \beta^{j k]} .
$$

This expression is still covariant. In DFT we may equally well solve the strong constraint by setting the conventional derivatives to zero, $\partial_{i}=0$, keeping the winding derivatives $\tilde{\partial}^{i}$. This corresponds to a T-duality inversion in all directions. The $R$ flux now reads

$$
R^{i j k}=3 \tilde{\partial}^{[i} \beta^{j k]} .
$$


Note that that the $R$ flux together with the $Q$-flux satisfies the following Bianchi identity $[27,30]^{2}$

$$
\tilde{\nabla}^{[i} R^{j k l]}=0,
$$

which reads explicitly

$$
4 \tilde{\partial}^{[i} R^{j k l]}+4 \beta^{p[i} \partial_{p} R^{j k l]}+6 Q_{p}{ }^{[i j} R^{k l] p}=0 .
$$

Via DFT or via field redefinitions we can also formulate an effective action for non-geometric string backgrounds. This new effective action is particularly useful in case the standard $b$-field and the standard are not well-defined quantities, as it is generically the case for non-geometric string background spaces. Specifically, the DFT action in terms of the new fields takes the following schematic form:

$$
\begin{aligned}
S_{\mathrm{DFT}}(\tilde{g}, \beta, \tilde{\phi}) & =\int d x d \tilde{x} \sqrt{|\tilde{g}|} e^{-2 \tilde{\phi}}\left[\mathscr{R}(\tilde{g}, \partial)+\mathscr{R}\left(\tilde{g}^{-1}, \tilde{\partial}\right)\right. \\
& \left.-\frac{1}{4} Q^{2}-\frac{1}{12} R^{i j k} R_{i j k}+4\left((\partial \tilde{\phi})^{2}+(\tilde{\partial} \tilde{\phi})^{2}\right)+\ldots\right]
\end{aligned}
$$

There are two Einstein-Hilbert terms: one based on the conventional derivative $\partial_{i}$, and one based on the winding derivatives $\tilde{\partial}^{i}$, where the inverse metric $\tilde{g}^{i j}$ plays the role of the usual metric, and so works consistently with the lower indices of the winding coordinates $\tilde{x}_{i}$. Even though the first Einstein-Hilbert term is manifestly invariant under $x$ diffeomorphisms $x^{i} \rightarrow x^{i}-\xi^{i}(x)$, and the second Einstein-Hilbert term is manifestly invariant under $\tilde{x}$ diffeomorphisms $\tilde{x}_{i} \rightarrow \tilde{x}_{i}-\tilde{\xi}_{i}(\tilde{x})$, the invariance of the full action as written in (2.14) is not manifest for either of them. The reason is that in the full DFT the parameters $\xi^{i}$ and $\tilde{\xi}_{i}$ can a priori depend both on $x$ and $\tilde{x}$. Moreover, as mentioned above, $Q$ is not a tensor and therefore the $Q^{2}$ term is not separately diffeomorphism invariant. As we have shown, in our formalism precisely half of the gauge symmetries can be made manifest, here the diffeomorphisms parameterized by $\xi^{i}$, by introducing a novel tensor calculus. The $Q$ can then be interpreted as the antisymmetric part of the 'dual' connection coefficients, so that the $Q^{2}$ term is just part of an extended dual Einstein-Hilbert term.

In summary, we finally have introduced the following chain of three T-duality transformations relating four different types of geometrical and non-geometrical spaces:

$$
H_{a b c} \stackrel{T_{x}}{\longrightarrow} f_{b c}^{a} \stackrel{T_{y}}{\longrightarrow} Q_{c}{ }^{a b} \stackrel{T_{z}}{\longrightarrow} R^{a b c} .
$$

In the next section we will discuss that depending on the considered duality frame, the phase space of the theory is described by a non-commutative and non-associative twisted Poisson structure, namely either for the coordinates or for the dual coordinates.

\subsection{Non-commutative and non-associative geometry from fluxes}

In the following we will relate the $Q, R$-fluxes to the non-commutative and non-associate geometry structure, which is present in non-geometric string backgrounds. Concretely, a noncommutative algebra for the string coordinates emerges for the $Q$-flux spaces, and the $R$-flux backgrounds even lead to a non-associative algebra structure. ${ }^{3}$ It is the non-geometric flux $R=\tilde{\partial} \beta$,

\footnotetext{
${ }^{2}$ This Bianchi identities was also derived by a different method in [31].

${ }^{3}$ These non-commutative and non-associatives structures also appeared in more mathematics oriented literature $[32,33,34,35,36]$, where twisted K-theory is applied to characterize these kind of non-geometric backgrounds with D-branes and $B$-fields.
} 
which corresponds to parameter that controls the violation of the Jacobi identity, i.e. to the deformation parameter of the non-associative algebra of the $R$-flux backgrounds.

Let us therefore try to relate the geometrical objects $\beta^{m n}, Q_{m}{ }^{n l}$ and $R^{m n l}$ discussed in this paper to the deformation parameters of the associated non-commutative respective non-associative algebras.

\subsubsection{Open strings}

One first relevant observation in this context is that these objects are closely related to the non-commutative open string geometry on these spaces. Consider frame (A) with a $b$-field on the two-dimensional fibre torus $\mathscr{F}=T_{x, y}^{2}$ :

$$
b=b(z) d x \wedge d y
$$

The corresponding $H$-field is given as

$$
H=\frac{\partial b(z)}{\partial z} d x \wedge d y \wedge d z
$$

One can easily convince oneself that the open string metric $g_{\text {open }}$ and the open string non-commutativity deformation $\theta$ (see [5]) parameter correspond to the dual metric $\tilde{g}$ and the bi-vector $\beta$, which will be defined in the following. Note that $\tilde{g}_{\text {open }}$ is just the open string metric in the limit where gravity is decoupled from the D2-brane world volume. Concretely, for D2-branes that are wrapped around the torus fibre of the $H$ flux space, one obtains the following equal-time commutator for the open string coordinates (at the location $\sigma=0, \pi$ of the D2-brane):

$$
\left[X^{m}(\tau), X^{n}(\tau)\right]_{\mathrm{open}}=\beta^{m n} .
$$

This defines a so-called Poisson structure in analogy to the momentum algebra of a point particle moving in a (constant) magnetic field.

\subsubsection{Closed strings}

We will start the discussion in the duality frame $\mathrm{C}$, namely we will describe the non-commutative geometry of closed strings moving in the non-geometric $Q$ flux background. A first guess could be that the non-commutativity is again directly related to the bi-vector $\beta$, leading to the same algebra (2.18) as for the open strings. However, this will not be quite correct: as discussed in $[11,14]$, only an extended closed string which is wrapped $\tilde{p}^{k}$ times around the base of the fibration is sensitive to the global ill-definedness of the two-dimensional fibre torus. As a result the fibre geometry becomes non-commutative with non-commutativity deformation parameter given in terms of the winding number $\tilde{p}^{k}$ :

$$
\left[X^{m}(\tau, \sigma), X^{n}(\tau, \sigma)\right]_{\text {closed }} \sim \varepsilon_{k}^{m n} \tilde{p}^{k} .
$$

In view of this result, we propose the following integral relation between the non-geometric $Q$ flux and the closed string non-commutativity [30]:

$$
\left[X^{m}(\tau, \sigma), X^{n}(\tau, \sigma)\right]_{\mathrm{closed}}=\oint_{C_{k}} Q_{k}^{m n}(X) d X^{k},
$$




\begin{tabular}{|c||c|c|}
\hline Flux & Commutators & Three-brackets \\
\hline \hline Frame A: $H$-flux & {$[\tilde{X}, \tilde{Y}] \simeq H \tilde{p}_{z}$} & {$[\tilde{X}, \tilde{Y}, \tilde{Z}] \simeq H$} \\
Frame B: $f$-flux & {$[X, \tilde{Y}] \simeq f \tilde{p}_{z}$} & {$[X, \tilde{Y}, \tilde{Z}] \simeq f$} \\
Frame C: $Q$-flux & {$[X, Y] \simeq Q \tilde{p}_{z}$} & {$[X, Y, \tilde{Z}] \simeq Q$} \\
Frame D: $R$-flux & {$[X, Y] \simeq R p_{z}$} & {$[X, Y, Z] \simeq R$} \\
\hline
\end{tabular}

Table 1: Non-vanishing commutators and three-brackets in the four flux backgrounds.

where $C_{k}$ is a non-trivial homology base cycle, around which the closed string is wrapped $\tilde{p}^{k}$ times. In the case of constant flux $Q_{k}{ }^{m n}=Q \varepsilon_{k}{ }^{m n}$ and $C_{k}=S^{1}$ one gets

$$
\left[X^{m}(\tau, \sigma), X^{n}(\tau, \sigma)\right]_{\mathrm{closed}}=\oint_{C_{k}} Q_{k}{ }^{m n} d X^{k}=2 \pi Q \varepsilon_{k}{ }^{m n} \tilde{p}^{k},
$$

in agreement with (2.19). So in more general terms, the right hand side of the commutator is given in terms of the Wilson line operator of the gauge connection $Q$. Although the $Q$-flux not being a covariant object, it is conceivable that the Wilson line operator is indeed a covariant object under coordinate transformations.

Next we turn to the duality frame $\mathrm{D}$, and we finally discuss the $R$ flux background obtained by a T-duality transformation, $X^{k} \leftrightarrow \tilde{X}_{k}$, in the $k^{\text {th }}$ direction from the previous case. The corresponding closed string background becomes non-associative, as discussed in [10] in the context of the $S U(2)$ Wess-Zumino-Witten model, and investigated in [12] by the computation of conformal field theory amplitudes in the chain of T-dual $H, f, Q, R$-backgrounds leading to a non-associative algebra of closed string vertex operators. So it is quite natural to conjecture that the non-geometric flux $R$ corresponds to the parameter that controls the violation of the Jacobi identity, i.e. to the deformation parameter of the non-associative algebra of the $R$ flux backgrounds:

$$
\left[\left[X^{m}(\tau, \sigma), X^{n}(\tau, \sigma)\right], X^{k}(\tau, \sigma)\right]_{\text {closed }}+\text { perm. }=R^{m n k} .
$$

Note that this non-associativity relation can be at least formally derived from the commutator (2.21) by using the Heisenberg commutation relation $\left[X^{k}, p^{k}\right]=i$ in the $k^{\text {th }}$ direction.

By T-duality we can even include the geometrical spaces (Frames A and B) in our discussion. For the $H$-flux background as well as for the geometrical $f$-flux the coordinates are still commutative and associative. For these backgrounds, the non-commutativity however shows up when including the dual coordinates of these spaces. Without going into the details of all possible T-duality transformations, we summarize the results in table 1 , where the commutation relations between coordinates $X^{a}$ and their duals $\tilde{X}^{a}$ are listed.

\section{3. (Non)-geometric backgrounds with elliptic monodromies and their (a)symmetric orbifold CFT's}

In this chapter we will derive the algebraic structures, discussed in the previous section, within a concrete class of string backgrounds, namely non-geometric backgrounds with elliptic monodromies. We will first show the derivation of the non-commutative closed string geometry 
using a twisted mode expansion for the coordinates, that reflects the monodromy properties of the non-geometric string background [11]. After that we will provide an exact non-commutative asymmetric orbifold CFT, that is valid at the orbifold point of the non-geometric background [14].

\subsection{Non-commutativity from elliptic monodromies and twisted closed string mode expansion}

So let us start to discuss torus fibrations with elliptic monodromies and their non-commutativity. Here, we can consider a chain of two T-dualities leading to three different dualities frames ${ }^{4}$

(Frame B): The $f$-flux space:

We consider again a twisted three-torus $T^{3}$, being a torus $T^{2}$ fibered over $S^{1}$ with coordinates $X^{1}, X^{2}$ on the fiber and $\mathbb{X}$ on the base. The metric of the total space is of the of the following form:

$$
d s^{2}=\frac{1}{\tau_{2}(\mathbb{X})}\left|d X^{1}+\tau(\mathbb{X}) d X^{2}\right|^{2}+d \mathbb{X}^{2}
$$

with the complex structure $\tau=\tau_{1}+i \tau_{2}$ of the fiber being, in general, a non-trivial function of the base space coordinate. An important class of torus fibrations is given by elliptic monodromies, which act on the toroidal coordinates as rotations. For example, consider the monodromy corresponding to a $\mathbb{Z}_{4} \subset O(2 ; \mathbb{Z})$ rotation:

$$
\left(\begin{array}{l}
X^{1} \\
X^{2}
\end{array}\right) \rightarrow\left(\begin{array}{c}
X^{2} \\
-X^{1}
\end{array}\right)
$$

resulting in an $S L(2 ; \mathbb{Z})$-transformation of the complex structure of the fiber:

$$
\tau(\mathbb{X}) \rightarrow-1 / \tau(\mathbb{X})
$$

As we will discuss in the next subsection, for $\tau(\mathbb{X})=i$, the complex structure is a fixed point of the above transformation. At this point in moduli space, the fibered torus admits an exact CFT description in terms of a freely-acting $\mathbb{Z}_{4}$-orbifold, corresponding to the minimum of the ScherkSchwarz potential for the complex structure.

One can diagonalize the $\mathbb{Z}_{4}$ rotation (3.2) by introducing complex coordinates $Z=\frac{1}{\sqrt{2}}\left(X^{1}+\right.$ $\left.i X^{2}\right)$ and, hence, obtain the following twisted boundary conditions:

$$
Z(\tau, \sigma+2 \pi)=e^{2 \pi i \theta} Z(\tau, \sigma),
$$

with the angle $\theta$ depending on the winding number (dual momentum) $\tilde{p}^{\mathbb{X}}$ in the $S^{1}$-direction:

$$
\theta=-f \tilde{p}^{\mathbb{X}} \quad, \quad \text { with } f \in \frac{1}{4}+\mathbb{Z} .
$$

Even though the complex structure $\tau(\mathbb{X})$ is a non-trivial function of the base coordinate $\mathbb{X}$ and the $\sigma$-model is, in general, non-linear, one could still write down a mode expansion for the fiber coordinates subject to the twisted boundary conditions (3.4), with the understanding that the result

\footnotetext{
${ }^{4}$ For this class of models there is no T-duality transformation that brings us to a background with only non-trivial $H$-flux (frame A).
} 
is only a lowest-order approximation in the $\omega$ - or $H$-flux (and in $\alpha^{\prime}$ ). Introducing the usual leftand right-moving coordinates $Z_{L, R}$, one obtains:

$$
\begin{aligned}
& Z_{L}(\tau+\sigma)=\frac{i}{\sqrt{2}} \sum_{k \in \mathbb{Z}} \frac{\alpha_{k-\theta}}{k-\theta} e^{-i(k-\theta)(\tau+\sigma)}, \\
& Z_{R}(\tau-\sigma)=\frac{i}{\sqrt{2}} \sum_{k \in \mathbb{Z}} \frac{\tilde{\alpha}_{k+\theta}}{k+\theta} e^{-i(k+\theta)(\tau-\sigma)} .
\end{aligned}
$$

Similar expansions hold for the complex conjugates, $\bar{Z}_{L, R}$. The usual quantization procedure then leads to the familiar bosonic oscillator algebra for the complex-conjugate Fourier modes:

$$
\left[\alpha_{k-\theta}, \bar{\alpha}_{\ell-\theta}\right]=(k-\theta) \delta_{k, \ell} .
$$

Explicit calculation then yields the equal- $\tau$ commutation relations for the coordinates ${ }^{5}$ :

$$
\left[Z_{L}(\tau, \sigma), \bar{Z}_{L}\left(\tau, \sigma^{\prime}\right)\right]=\frac{1}{2} \sum_{k \in \mathbb{Z}} \frac{e^{-i(k-\theta)\left(\sigma-\sigma^{\prime}\right)}}{k-\theta} \equiv \frac{1}{2} \Theta\left(\sigma-\sigma^{\prime}, \tilde{p}^{\mathbb{X}}\right)
$$

For the right-moving coordinates, a similar calculation yields the same function $\Theta$, but with the opposite sign:

$$
\left[Z_{R}(\tau, \sigma), \bar{Z}_{R}\left(\tau, \sigma^{\prime}\right)\right]=-\frac{1}{2} \Theta\left(\sigma-\sigma^{\prime}, \tilde{p}^{\mathbb{X}}\right) .
$$

In order to obtain a local result, one should carefully investigate the limit $\sigma \rightarrow \sigma^{\prime}$. Since in this limit, the series representation (3.8) is naively divergent, it has to be defined through analytic continuation. To this end, upon introducing the complex variable $z=e^{i\left(\sigma^{\prime}-\sigma\right)}$, the function $\Theta$ can be neatly represented in terms of hypergeometric functions:

$$
\Theta\left(\sigma-\sigma^{\prime}, \tilde{p}^{\mathbb{X}}\right)=-\frac{z^{-\theta}}{\theta}\left[{ }_{2} F_{1}(1,-\theta ; 1-\theta ; z)+{ }_{2} F_{1}\left(1, \theta ; 1+\theta ; z^{-1}\right)-1\right],
$$

which can then be analytically continued to $z \rightarrow 1$. The final result for $\Theta$ is given in terms of elementary functions:

$$
\Theta\left(\tilde{p}^{\mathbb{X}}\right) \equiv\left\{\begin{array}{cc}
-\pi \cot (\pi \theta), & \theta \notin \mathbb{Z} \\
0, & \theta \in \mathbb{Z}
\end{array},\right.
$$

modulo the discontinuity at $\theta \in \mathbb{Z}$, arising from the subtraction of the zero mode in (3.8).

As expected for a geometric background, the twisted torus leads to commutative coordinates:

$$
\left[X^{1}(\tau, \sigma), X^{2}(\tau, \sigma)\right]=0
$$

where we have expressed the commutation relations in terms of the original coordinates $X^{1}, X^{2}$ of the torus fiber.

(Frame C): The $Q$-flux space:

\footnotetext{
${ }^{5}$ Strictly speaking, the commutation algebra should include the metric factor $G^{z \bar{z}}$, but this is immaterial for our present discussion and we will simply suppress it.
} 
Performing a T-duality in the $X^{1}$-direction, one is lead to a non-commutative coordinate algebra. Indeed, introducing the dual coordinate $\tilde{X}^{1}=X_{L}^{1}-X_{R}^{1}$, one obtains:

$$
\left[\tilde{X}^{1}(\tau, \sigma), X^{2}(\tau, \sigma)\right]=\left[X_{L}^{1}(\tau, \sigma), X_{L}^{2}(\tau, \sigma)\right]-\left[X_{R}^{1}(\tau, \sigma), X_{R}^{2}(\tau, \sigma)\right]=i \Theta\left(\tilde{p}^{\mathbb{X}}\right) .
$$

The same commutation algebra arises by imposing from the very beginning the following asymmetric boundary conditions:

$$
\begin{aligned}
& Z_{L}(\tau, \sigma+2 \pi)=e^{2 \pi i \theta} Z_{L}(\tau, \sigma), \\
& Z_{R}(\tau, \sigma+2 \pi)=e^{-2 \pi i \theta} Z_{R}(\tau, \sigma),
\end{aligned}
$$

which are induced by the following monodromy transformation now acting on the Käbler parameter $\rho(\mathbb{X})$ of the two-torus in the following way:

$$
\rho(\mathbb{X}) \rightarrow-1 / \rho(\mathbb{X}) .
$$

These transformations are highly reminiscent of an asymmetric orbifold. The first line in eq. (3.14) corresponds to the $\mathbb{Z}_{4}$-action:

$$
X_{L}^{1} \rightarrow X_{L}^{2} \quad, \quad X_{L}^{2} \rightarrow-X_{L}^{1},
$$

whereas the second line now gives for the right-moving sector:

$$
X_{R}^{1} \rightarrow-X_{R}^{2} \quad, \quad X_{R}^{2} \rightarrow X_{R}^{1} .
$$

These asymmetric rotations define the following monodromy element $g$ of the $O(2,2 ; \mathbb{Z})$ T-duality group:

$$
g=\left(\begin{array}{cccc}
0 & 0 & 0 & 1 \\
0 & 0 & -1 & 0 \\
0 & 1 & 0 & 0 \\
-1 & 0 & 0 & 0
\end{array}\right) .
$$

Notice that $g$ is no longer an element of the geometric subgroup $G L(2 ; \mathbb{Z})$.

Now using the corresponding asymmetric mode expansion one obtains for the commutator of the torus coordinates: 6

$$
\left[X^{1}(\tau, \sigma), X^{2}(\tau, \sigma)\right]=i \Theta\left(\tilde{p}^{\mathbb{X}}\right) .
$$

The arguments presented above, hence, support the following conclusion. In stringy nongeometric compactifications, non-commutativity for the closed string coordinates can arise whenever the monodromy matrix $g \in O(N, N ; \mathbb{Z})$ is not an element of the geometric subgroup $G L(N ; \mathbb{Z})$. Furthermore, for special points in the moduli space of the theory, an exact CFT description in terms of freely-acting asymmetric orbifolds may exist for such non-geometric models. Of course, the construction of asymmetric orbifolds is not automatic. In general, modular invariance severely constrains the space of consistent asymmetric orbifold vacua. In the next subsection we will present explicit constructions of freely-acting asymmetric toroidal orbifolds and show how noncommutativity arises in these setups. In particular, our results will be exact to all orders in $\alpha^{\prime}$.

\footnotetext{
${ }^{6}$ More general asymmetric rotations are possible for other choices of $\theta_{L}$ and $\theta_{R}$, provided they are compatible with modular invariance. In this case, the algebra of coordinates becomes $\left[X^{1}, X^{2}\right]=\frac{i}{2}\left\{\Theta\left(\theta_{L}\right)-\Theta\left(\theta_{R}\right)\right\}$.
} 
(Frame D): The R-flux space:

The R-flux space is reached by performing a T-duality transformation in the $\mathbb{X}$-direction. Although we cannot write down a local metric for this background, one can perform the T-duality transformation on the level of the non-commutative algebra by simply replacing the winding number, i.e. the dual momentum number $\tilde{p}^{\mathbb{X}}$ by the T-dual KK momentum number $p^{\mathbb{X}}$. Then the algebra of two-torus coordinates becomes:

$$
\left[X^{1}(\tau, \sigma), X^{2}(\tau, \sigma)\right]=i \Theta\left(p^{\mathbb{X}}\right) \quad \text { with } \quad \Theta\left(p^{\mathbb{X}}\right)=\pi \cot \left(\pi f p^{\mathbb{X}}\right) .
$$

Using the the Heisenberg commutation relation $\left[\mathbb{X}, p^{\mathbb{X}}\right]=i$ and linearizing the cot-function one finally obtains the non-associative structure

$$
\left.\left[\left[X^{1}(\tau, \sigma), X^{2}(\tau, \sigma)\right], \mathbb{X}(\tau, \sigma)\right]\right]=f \pi^{2}
$$

\subsection{Non-commutativity in asymmetric orbifold CFT's}

The fully consistent study of non-commutative effects in string theory necessitates a treatment that is exact to all orders in $\alpha^{\prime}$. A natural candidate is provided by a class of freely-acting asymmetric $\mathbb{Z}_{N}$-orbifolds. The advantage of considering such backgrounds is that the corresponding worldsheet CFT is locally free, allowing us to obtain exact mode expansions for the internal coordinates and their commutators, $\left[X^{I}, X^{J}\right]$. We will start with the construction of a simple class of freely-acting asymmetric orbifold models. To illustrate the consistency of the models, we will explicitly display their modular invariant partition function. These models should be considered as special solvable points in the moduli space of more general, non-geometric $Q$-flux backgrounds. Hence, these results will be exact to all orders in $\alpha^{\prime}$ and to all orders in the (quantized) value of the flux ${ }^{7}$.

The constructions [14] we present will be Type II (freely-acting) asymmetric orbifold models with $4 \leq \mathscr{N}_{4}<8$ spacetime supersymmetry, compactified on $\left(S^{1} \times T^{5}\right) / \mathbb{Z}_{N}$. The action of the $\mathbb{Z}_{N}$ on $T^{5}$ will be specified below. Let us also note that the restriction to Type II theories is only a convenient choice, as our results can be extended to Heterotic theories in a straightforward fashion.

We will start our discussion by considering an $N$-dimensional torus $T^{N} \subseteq T^{5}$, that is locally factorized from an $S^{1}$-circle of radius $R$. We will then proceed by defining the action of $\mathbb{Z}_{N}$ on this manifold and derive various consistency conditions, such that the theory is a well-defined asymmetric orbifold. In fact, in what concerns the $T^{N}$, we will restrict our attention to asymmetric orbifolds where the $\mathbb{Z}_{N}$ acts non-trivially only on the left-moving degrees of freedom. To this end, the discussion in this section refers to the left-moving worldsheet fields. It is then easy to extend the construction to the right-moving sector in order to define symmetric orbifolds as well.

Let us take the $N$-dimensional torus $T^{N}$ to be parametrized by coordinates $X^{I}$, with $I=$ $1, \ldots, N \leq 5$ and, further denote the coordinate associated to the $S^{1}$-circle by $\mathbb{X}$. We will restrict ourselves to the case where the orbifold acts as a permutation $P$ (including a possible 'reflection') only on the left-moving coordinates of $T^{N}$, while leaving the right-movers invariant. Furthermore, in order to eliminate fixed points and generate a free action, we will couple this to a shift both in

\footnotetext{
${ }^{7}$ Contrary to the case of non-freely acting orbifolds, in which the internal space is singular at fixed points, the orbifolds we construct are free of such singularities, due to their freely-acting nature.
} 
the momenta and windings of the $S^{1}$-direction in the orbifold basis. The orbifold element can be expressed, hence, as:

$$
g=e^{2 \pi i Q_{L}} \delta
$$

where $Q_{L}$ is the generator of permutations (with possible reflections) of the left-moving $T^{N}$ coordinates and $\delta$ is an order $1 / N$-shift in the $S^{1}$-direction. Of course, since we are considering an asymmetric orbifold, the construction can only take place at special points in the moduli space of the theory, where the CFT factorizes and purely left-moving lattice isometries exist. For simplicity, the models will be constructed at the fermionic point, where chiral bosons can be defined through fermionization.

We shall now define the orbifold action on the left-moving $X^{I}$-coordinates of $T^{N}$. Consider the permutations (with a possible reflection) defined by the matrix:

$$
P_{I J}(\varepsilon)=\left(\begin{array}{cccccc}
0 & 1 & 0 & \ldots & 0 & 0 \\
0 & 0 & 1 & \ldots & 0 & 0 \\
\vdots & \vdots & \vdots & \ldots & \vdots & \vdots \\
0 & 0 & 0 & \ldots & 0 & 1 \\
\varepsilon & 0 & 0 & \ldots & 0 & 0
\end{array}\right)_{I J}=\delta_{I, J-1}+\varepsilon \delta_{I, J+N-1}, \text { where } \varepsilon= \pm 1
$$

where we display the orbifold action on the relevant coordinates only. Notice that in the absence of reflection $(\varepsilon=+1)$, this corresponds to a $\mathbb{Z}_{N}$-orbifold. On the other hand, including a non-trivial reflection $(\varepsilon=-1)$, leads to an enhanced $\mathbb{Z}_{2 N}$.

We can now pick a basis that diagonalizes $P(\varepsilon)$ as follows. If $\left\{\lambda_{I}\right\}$ is the set of eigenvalues of $P(\varepsilon)$, then we can define the linear combinations:

$$
Z^{I}=\frac{1}{\sqrt{N}} \sum_{J=1}^{N}\left(\lambda_{I}\right)^{J-1} X^{J}, \text { with } I=1, \ldots, N
$$

which are the eigenvectors of $P(\varepsilon)$ with eigenvalues:

$$
\lambda_{I}(\varepsilon)=e^{2 \pi i(I-1+v) / N}, \text { with } v(\varepsilon)=\left\{\begin{array}{cc}
0 & , \text { for } \varepsilon=+1 \\
1 / 2 & , \text { for } \varepsilon=-1
\end{array} .\right.
$$

Notice that the new coordinates $Z^{I}$ can be either real or complex depending on the corresponding eigenvalue $\lambda_{I}$.

We now fermionize the $T^{N}$ coordinates of the original basis as:

$$
i \partial X^{I}=i y^{I} \omega^{I},
$$

where $y^{I}, \omega^{I}$ are real (auxiliary) free fermions. The change of basis is then defined by the unitary matrix:

$$
U_{I J}(\varepsilon)=\frac{1}{\sqrt{N}}\left(\begin{array}{cccccc}
1 & \lambda_{1} & \lambda_{1}^{2} & \ldots & \lambda_{1}^{N-2} & \lambda_{1}^{N-1} \\
1 & \lambda_{2} & \lambda_{2}^{2} & \ldots & \lambda_{2}^{N-2} & \lambda_{2}^{N-1} \\
\vdots & \vdots & \vdots & \ldots & \vdots & \vdots \\
1 & \lambda_{N} & \lambda_{N}^{2} & \ldots & \lambda_{N}^{N-2} & \lambda_{N}^{N-1}
\end{array}\right)_{I J}=\frac{1}{\sqrt{N}} e^{2 \pi i(I-1+v)(J-1) / N}
$$


Note that the $\varepsilon$-dependence of this matrix arises only implicitly, through the appearance of $v=$ $\frac{1}{4}(1-\varepsilon)$ in the eigenvalues. Explicitly, the change of basis on the coordinates and their fermionic (worldsheet) superpartners is:

$$
\begin{aligned}
Z^{I} & =U_{J}^{I}(\varepsilon) X^{J}, \\
\Psi^{I} & =U_{J}^{I}(\varepsilon) \psi^{J} .
\end{aligned}
$$

In this basis, the orbifold acts simply as a rotation by a phase:

$$
\begin{aligned}
Z_{L}^{I} & \rightarrow \lambda_{I}(\varepsilon) Z_{L}^{I}, \\
\Psi_{L}^{I} & \rightarrow \lambda_{I}(\varepsilon) \Psi_{L}^{I} .
\end{aligned}
$$

The action of the orbifold on the auxiliary free fermions is:

$$
\begin{gathered}
y^{I} \rightarrow P_{J}^{I}(\varepsilon) y^{J}, \\
\omega^{I} \rightarrow P_{J}^{I}(1) \omega^{J} .
\end{gathered}
$$

Notice that the $\omega$-fermions always transform under the orbifold as a pure permutation $(\varepsilon=1)$, in order for their product $y^{I} \omega^{I}$ to correctly represent the orbifold action on the bosons $\partial X^{I}$. Hence, the correct basis redefinition for the auxiliary free fermions is:

$$
\begin{aligned}
Y^{I} & =U_{J}^{I}(\varepsilon) y^{I}, \\
W^{I} & =U^{I}{ }_{J}(1) \omega^{J} .
\end{aligned}
$$

It is, again, crucial that the $\omega$-fermions change basis as if there was no reflection $(\varepsilon=+1$ or $v=0)$.

In the new basis $\left\{Z^{I}, \Psi^{I}\right\}$, the coordinates and their fermionic superpartners generically arise as complex worldsheet fields. Complex eigenvalues always come in pairs $\left(\lambda^{*}=\varepsilon \lambda^{N-1}\right)$, so that we can group the associated coordinates such that $Z^{I}, Z^{J}$ are complex conjugate to each other and similarly for $\Psi^{I}, \Psi^{J}$. This complexification is, in fact, crucial for the consistency of our asymmetric construction and it will permit us to represent the partition function of the theory in terms of simple level-1 characters.

It is important to note that the $Z^{I}$ are not fermionized into the simple product $Y^{I} W^{I}$ but, rather, into a linear combination of fermion bilinears:

$$
\begin{aligned}
i \partial Z^{I} & =i \sum_{J=1}^{N} U_{J}^{I}(\varepsilon)\left(U^{-1}\right)^{J}{ }_{K}(\varepsilon)\left(U^{-1}\right)^{J}{ }_{L}(1) Y^{K} W^{L} \\
& =\frac{i}{\sqrt{N}} \sum_{K, L=1}^{N} \delta_{(I-K-L+1) \bmod N, 0} Y^{K} W^{L},
\end{aligned}
$$

where the Kronecker $\delta$-function restricts the sum to those $K, L$ which satisfy the constraint ( $I-$ $K-L+1) \bmod N=0$. This constraint picks up precisely the combinations that correctly reproduce the $\mathbb{Z}_{N}$-transformation of $\partial Z^{I}$, as expected.

The above fermionization of the coordinates is only consistent at the fermionic point in moduli space. In our conventions, this is a square lattice $G_{I J}=r^{2} \delta_{I J}$, with $r=1 / \sqrt{2}$ being the fermionic radius. The orbifold action typically introduces additional constraints. First of all, a non-trivial 
requirement is that the orbifold action $P(\varepsilon)$ must be a symmetry of the local $\mathscr{N}=1$ superconformal theory (SCFT) on the worldsheet. It is straightforward to check that both $T_{B}$ and $T_{F}$ of the internal SCFT are invariant, since at the fermionic point the orbifold acts crystallographically:

$$
\begin{aligned}
& T^{\text {int }}(z)=-r^{2} \sum_{I=1}^{N}\left(\partial X^{I}\right)^{2}-\frac{r^{2}}{2} \sum_{I=1}^{N} \psi_{L}^{I} \partial \psi_{L}^{I}+\ldots=-r^{2} \widehat{\sum_{I, J}}\left(\partial Z^{I} \partial Z^{J}-\frac{1}{2} \Psi_{L}^{I} \partial \Psi_{L}^{J}\right)+\ldots, \\
& T_{F}^{\text {int }}(z)=i \sqrt{2} r^{2} \sum_{I=1}^{N} \psi_{L}^{I} \partial X^{I}+\ldots=i \sqrt{2} r^{2} \widehat{\sum}_{I, J} \Psi_{L}^{I} \partial Z^{J}+\ldots
\end{aligned}
$$

where $\widehat{\sum}$ stands for the sum subject to the constraint $I+J \in N \mathbb{Z}+2(1-v)$ and the dots denote contributions of the remaining coordinates in $T^{5}$ that are not transformed, as well as the contributions due to the (super-)ghosts and the $S^{1}$ (super-)coordinate. Of course, at the fermionic point, $T_{B}$ and $T_{F}$ can be realized entirely in terms of the free fermions $\psi^{I}, y^{I}$ and $\omega^{I}$ and similar relations hold.

We can now write the modular invariant partition function of the model (up to an overall $\tau_{2}$ power) in the following form:

$$
Z=\frac{1}{\eta^{12} \bar{\eta}^{12}} R \sum_{\tilde{m}, n \in \mathbb{Z}} e^{-\frac{\pi R^{2}}{\tau_{2}}|\tilde{m}+\tau n|^{2}} Z_{L}\left[\begin{array}{l}
h \\
g
\end{array}\right](\tau) \tilde{Z}_{R}(\bar{\tau}) \Gamma_{(5,5)}\left[\begin{array}{l}
h \\
g
\end{array}\right](\tau, \bar{\tau})
$$

The orbifold twist variables are defined as follows:

$$
h=\frac{2 \tilde{p}^{\mathbb{X}}}{N}, g=\frac{2 p^{\mathbb{X}}}{N},
$$

where $\tilde{p}^{\mathbb{X}}, p^{\mathbb{X}} \in \mathbb{Z}$ are the winding quantum numbers around the $S^{1}$, as each of the worldsheet coordinates $\sigma^{1}, \sigma^{2}$ encircle the two non-trivial cycles of the worldsheet torus. $Z_{R}\left[\begin{array}{l}h \\ g\end{array}\right]$ and $\tilde{Z}_{R}$ are the contributions of the left- and right- moving worldsheet fermion superpartners $\psi, \tilde{\psi}$, respectively and $\Gamma_{(5,5)}\left[\begin{array}{l}h \\ g\end{array}\right]$ is the $(5,5)$-lattice associated to the $T^{5}$. The exponential factor in (3.34) is the Lagrangian representation of the lattice partition function of the $S^{1}$ coordinate, $\mathbb{X}$. It is straightforward to check that the full partition function (3.34) is indeed modular invariant, up to an overall $\tau_{2}$-factor. Moreover, the partition function can be seen to arise as the modular-invariance preserving deformation of the ordinary toroidal compactification on $T^{5} \times S^{1}$. It is compatible with the constraints arising from higher-genus modular invariance and factorization and, hence, defines a class of consistent orbifold vacua.

So far, we have reviewed the construction of consistent freely-acting asymmetric $\mathbb{Z}_{N}$-orbifolds. One has to keep in mind that these backgrounds are to be considered as (smooth) orbifold limits of string compactifications with non-geometric $Q$-fluxes (T-folds). We are now ready to examine the algebra of commutators of the internal coordinates, subject to the $\mathbb{Z}_{N}$-orbifold boundary conditions (3.29). We will see that the asymmetric nature of the orbifold leads to a non-commutative algebra for the $T^{N}$ coordinates, the structure constants of which are nicely parametrized in terms of a flux matrix $F_{J}^{I}$. We will start by writing down the mode expansions directly in the basis $Z^{I}$ which diagonalizes the orbifold action. We restrict our attention to the left-movers:

$$
Z_{L}^{I}(\tau, \sigma)=\frac{i}{\sqrt{2}} \sum_{k \in \mathbb{Z}} \frac{\alpha_{k-\theta^{I}}^{I}}{k-\theta^{I}} e^{-i\left(k-\theta^{I}\right)(\tau+\sigma)},
$$


where the modes satisfy the standard commutation relations:

$$
\left[\alpha_{k-\theta^{I}}^{I}, \alpha_{\ell-\theta^{J}}^{J}\right]=\left(k-\theta^{I}\right) \delta_{k+\ell, 0}\left(U G^{-1} U^{T}\right)^{I J}
$$

Notice that:

$$
\left(U G^{-1} U^{T}\right)^{I J}=\frac{1}{r^{2}} \delta_{[I+J-2(1-v)] \bmod N, 0},
$$

is nothing but the inverse metric in the $Z^{I}$-basis. It is convenient to parametrize the orbifold action $P_{J}^{I}(\varepsilon)$ in terms of a real antisymmetric matrix $F_{J}^{I}$ :

$$
P_{J}^{I}=\left(e^{2 \pi F}\right)_{J}^{I} .
$$

The deformation angle $\theta^{I}=f^{I} \tilde{p}^{\mathbb{X}}$ is nothing but the $F$-flux eigenvalue $f^{I}$ times the winding number $\tilde{p}^{\mathbb{X}}$ along $S^{1}$ and is related to the eigenvalue $\lambda_{I}$ through:

$$
e^{2 \pi i \theta^{I}}=\lambda_{I}^{\tilde{p}^{\mathbb{X}}}=e^{2 \pi i f^{I} \tilde{p}^{\mathbb{X}}} .
$$

Explicitly, the matrix $F_{J}^{I}$ can be easily constructed from its eigenvalues, $\pm i f^{I}$ :

$$
f^{I}=\frac{(I-1+v)}{N} \quad, \quad \text { with } \quad I=1, \ldots,\left[\frac{N+1}{2}\right],
$$

and is given by the following expression:

$$
F_{J}^{I}=-\frac{2}{N} \sum_{K=1}^{\left[\frac{N+1}{2}\right]} f^{K} \sin \left(2 \pi f^{K}(I-J)\right)
$$

where the square brackets in the upper limit of the sum denote the integer part.

The commutation relations (3.37) should be supplemented with the 'reality condition':

$$
\left.\begin{array}{l}
\bar{\alpha}_{k-\theta^{I}}=\alpha_{-k+\theta^{I}}^{J} \\
\theta^{I}=-\theta^{J}
\end{array}\right\} \quad, \quad \text { if } \overline{Z^{I}}=Z^{J}
$$

which essentially reflects the fact that only commutators between complex conjugate pairs are nonvanishing.

We can now take a pair of coordinates and evaluate their equal-times commutator using (3.37), (3.38), and (3.43):

$$
\left[Z_{L}^{I}(\tau, \sigma), Z_{L}^{J}\left(\tau, \sigma^{\prime}\right)\right]=\frac{1}{2}\left(U G^{-1} U^{T}\right)^{I J} \sum_{k \in \mathbb{Z}} \frac{e^{-i\left(k-\theta^{I}\right)\left(\sigma-\sigma^{\prime}\right)}}{k-\theta^{I}} .
$$

Setting $z=e^{i\left(\sigma^{\prime}-\sigma\right)}$, we can express the series as the linear combination of hypergeometric functions in (3.10), which can then be analytically continued to $z \rightarrow 1$ :

$$
\Theta\left(\theta^{I}\right)=\left\{\begin{array}{ccc}
-\pi \cot \left(\pi \theta^{I}\right) & , & \theta^{I} \notin \mathbb{Z} \\
0 & , & \theta^{I} \in \mathbb{Z}
\end{array} .\right.
$$


Going back to the original basis, the commutation relations between the full coordinates $X=X_{L}+$ $X_{R}$ become $^{8}$ :

$$
\left.\left[X^{I}, X^{J}\right]\right|_{\sigma=\sigma^{\prime}}=\frac{1}{2} \Theta\left(i \tilde{p}^{\mathbb{X}} F\right)^{I J}
$$

Note that the argument of the function $\Theta$ in (3.46) is now a matrix and the easiest way to define it is through its eigenvalues. Since $F$ is at most five-dimensional and antisymmetric, it can always be expanded as:

$$
\Theta\left(i \tilde{p}^{\mathbb{X}} F\right)=\alpha F+\beta F^{3}
$$

with $\alpha, \beta$ complex coefficients that are determined by substituting in the eigenvalues of $F$, eq. (3.41).

In the cases $\mathbb{Z}_{2}, \mathbb{Z}_{3}$ and $\mathbb{Z}_{4}$, there is only a single complex eigenvalue ${ }^{9}$ and, hence, $\beta=0$. For the $\mathbb{Z}_{k}$ orbifold with $k=2,3,4$, the non-commutative algebra between the toroidal coordinates then takes the simple form:

$$
\left[X^{I}, X^{J}\right]=\frac{i}{2} k F^{I J} \Theta\left(\tilde{p}^{\mathbb{X}} / k\right)=\left\{\begin{array}{cc}
-\frac{i}{2} \pi k F^{I J} \cot \left(\frac{\pi \tilde{p}^{\mathbb{X}}}{k}\right), & \tilde{p}^{\mathbb{X}} \notin k \mathbb{Z} \\
0 & , \quad \tilde{p}^{\mathbb{X}} \in k \mathbb{Z}
\end{array} .\right.
$$

The case of $\mathbb{Z}_{2}$ is special, because the r.h.s. vanishes identically and the toroidal coordinates remain commutative. This is, essentially, due to the fact that the $\mathbb{Z}_{2}$-action can always be reduced to a reflection of a real coordinate and, hence, it does not really 'entangle' different coordinates.

It is instructive to comment on the structure of the general result (3.46). First of all, the stringy origin of the non-commutative behavior becomes manifest, by noticing that $\left[X^{I}, X^{J}\right]$ is always proportional to the string scale $\alpha^{\prime}$. Secondly, the explicit periodic dependence on the winding number $\tilde{p}^{\mathbb{X}}$ along the circle direction $\mathbb{X}$, implies that only when non-trivial winding modes along $S^{1}$ can be excited, can one probe the non-commutativity in the toroidal coordinates. This is consistent with the fact that, in the limit where the radius of $S^{1}$ becomes large $R / \alpha^{\prime} \gg 1$ and states carrying non-trivial winding charge become supermassive, the fibration of $T^{2}$ over $S^{1}$ trivializes and the non-commutative effects are screened.

\section{Conclusions}

As we have seen, non-geometric closed string backgrounds lead to spatial non-commutativity and non-associativity of the closed string coordinates. The associated algebras can be described by twisted Poisson structures in analogy to the momentum phase space of point particles in the field of magnetic monopoles. In string theory, the deformation parameters of the non-commutative/nonassociative algebras are given in terms of tensors ( $R$-flux) and connections ( $Q$-flux), which naturally arise in the context of doubled field theory and effective actions of non-geometric back-

\footnotetext{
${ }^{8}$ In this asymmetric orbifold, the right-moving coordinates are commutative. In this sense this model is different from the non-geometric flux background with elliptic monodromy, presented in section 3.1.

${ }^{9}$ In the $\mathbb{Z}_{4}$ case with $N=2, \varepsilon=-1$, the complex eigenvalue comes with multiplicity two because of the doubling of the orbifold action on $T^{4}$.
} 
grounds. Since closed strings are responsible for gravity, it is tempting to conjecture that the noncommmutative/non-associative closed string geometries will lead to a non-commmutative/nonassociative deformation of gravity. Furthermore, as these non-associative algebras go beyond matrix (quantum) mechanics, it is an interesting question, how to represent them, possibly by some kind of octonionic objects.

\section{Acknowledgments}

We would like to thank D. Andriot, R. Blumenhagen, C. Condeescu, A. Deser, I. Florakis, O. Hohm, M. Larfors, P. Patalong E. Plauschinn and F. Renneke for the very pleasant collaborations on the material presented in this paper. For very useful comments and discussions we would like to thank I. Bakas and T. Strobl. This work is supported by the DFG Transregional Collaborative Research Centre TRR 33 and the DFG cluster of excellence 'Origin and Structure of the Universe'.

\section{References}

[1] M. Grana, "Flux compactifications in string theory: A Comprehensive review," Phys. Rept. $\mathbf{4 2 3}$ (2006) 91 [hep-th/0509003].

[2] R. Blumenhagen, B. Körs, D. Lüst and S. Stieberger, "Four-dimensional String Compactifications with D-Branes, Orientifolds and Fluxes," Phys. Rept. 445 (2007) 1 [hep-th/0610327].

[3] C.-S. Chu and P.-M. Ho, Noncommutative open string and D-brane, Nucl.Phys. B550 (1999) 151-168, [hep-th/9812219].

[4] V. Schomerus, D-branes and deformation quantization, JHEP 9906 (1999) 030, [hep-th/9903205].

[5] N. Seiberg and E. Witten, String theory and noncommutative geometry, JHEP 9909 (1999) 032, [hep-th/9908142].

[6] F. Ardalan, H. Arfaei, and M. Sheikh-Jabbari, Mixed branes and M(atrix) theory on noncommutative torus, hep-th/9803067.

[7] C. M. Hull, “A Geometry for non-geometric string backgrounds," JHEP 0510, 065 (2005) [hep-th/0406102].

[8] J. Shelton, W. Taylor and B. Wecht, "Nongeometric flux compactifications," JHEP 0510 (2005) 085 [hep-th/0508133].

[9] A. Dabholkar and C. Hull, "Generalised T-duality and non-geometric backgrounds," JHEP 0605, 009 (2006) [hep-th/0512005].

[10] R. Blumenhagen, E. Plauschinn, "Nonassociative Gravity in String Theory?,” J. Phys. A A44 (2011) 015401. [arXiv:1010.1263 [hep-th]].

[11] D. Lüst, "T-duality and closed string non-commutative (doubled) geometry," JHEP 1012 (2010) 084. [arXiv:1010.1361 [hep-th]].

[12] R. Blumenhagen, A. Deser, D. Lüst, E. Plauschinn and F. Rennecke, "Non-geometric Fluxes, Asymmetric Strings and Nonassociative Geometry,” J. Phys. A A 44 (2011) 385401 [arXiv:1106.0316 [hep-th]].

[13] R. Blumenhagen, “Nonassociativity in String Theory," arXiv:1112.4611 [hep-th]. 
[14] C. Condeescu, I. Florakis and D. Lüst, "Asymmetric Orbifolds, Non-Geometric Fluxes and Non-Commutativity in Closed String Theory,” arXiv:1202.6366 [hep-th].

[15] E. Plauschinn, "Non-geometric fluxes and non-associative geometry," arXiv:1203.6203 [hep-th].

[16] C. Saemann and R. J. Szabo, “Groupoid Quantization of Loop Spaces,” arXiv:1203.5921 [hep-th].

[17] R. Jackiw, 3 - Cocycle in Mathematics and Physics, Phys.Rev.Lett. 54 (1985) 159-162.

[18] C. Klimcik, T. Strobl, “WZW - Poisson manifolds,” J. Geom. Phys. 43 (2002) 341-344. [math/0104189 [math-sg]].

[19] A. Alekseev, T. Strobl, “Current algebras and differential geometry,” JHEP 0503 (2005) 035. [hep-th/0410183].

[20] A. Kotov, P. Schaller, T. Strobl, "Dirac sigma models," Commun. Math. Phys. 260 (2005) 455-480. [hep-th/0411112].

[21] C.M. Hull, “Doubled Geometry and T-Folds,” JHEP 0707 (2007) 080 [hep-th/0605149].

[22] C. Hull and B. Zwiebach, "Double Field Theory," JHEP 0909 (2009) 099 [arXiv:0904.4664 [hep-th]].

[23] C. Hull and B. Zwiebach, "The Gauge algebra of double field theory and Courant brackets," JHEP 0909 (2009) 090 [arXiv:0908.1792 [hep-th]].

[24] O. Hohm, C. Hull and B. Zwiebach, "Background independent action for double field theory," JHEP 1007 (2010) 016 [arXiv:1003.5027 [hep-th]].

[25] O. Hohm, C. Hull and B. Zwiebach, JHEP 1008 (2010) 008 [arXiv:1006.4823 [hep-th]].

[26] D. Andriot, M. Larfors, D. Lüst and P. Patalong, "A ten-dimensional action for non-geometric fluxes," JHEP 1109 (2011) 134 [arXiv:1106.4015 [hep-th]].

[27] D. Andriot, O. Hohm, M. Larfors, D. Lüst and P. Patalong, "A geometric action for non-geometric fluxes," to appear in Phys.Rev.Lett., arXiv:1202.3060 [hep-th].

[28] P. Patalong, "Non-geometric Q-flux in ten dimensions," arXiv:1203.5127 [hep-th].

[29] M. Larfors, "Non-geometric fluxes in ten dimensions,"

[30] D. Andriot, O. Hohm, M. Larfors, D. Lüst and P. Patalong, "Non-Geometric Fluxes in Supergravity and Double Field Theory," arXiv:1204.1979 [hep-th].

[31] R. Blumenhagen, A. Deser, E. Plauschinn and F. Rennecke, "Palatini-Lovelock-Cartan Gravity Bianchi Identities for Stringy Fluxes,” arXiv:1202.4934 [hep-th].

[32] P. Bouwknegt and V. Mathai, D-branes, B fields and twisted K theory, JHEP 0003 (2000) 007, [hep-th/0002023].

[33] P. Bouwknegt, K. Hannabuss, and V. Mathai, Nonassociative tori and applications to T-duality, Commun.Math.Phys. 264 (2006) 41-69, [hep-th / 0412092 ].

[34] V. Mathai and J. M. Rosenberg, T duality for torus bundles with H fluxes via noncommutative topology, Commun.Math.Phys. 253 (2004) 705-721, [hep-th/ 0401168 ].

[35] V. Mathai and J. M. Rosenberg, On Mysteriously missing T-duals, H-flux and the T-duality group, hep-th/0409073.

[36] J. Brodzki, V. Mathai, J. M. Rosenberg, and R. J. Szabo, Noncommutative correspondences, duality and D-branes in bivariant K-theory, Adv.Theor.Math.Phys. 13 (2009) 497-552, [arXiv:0708.2648]. 